\title{
Coulomb-hadron phase factor and spin phenomena in a wide region of transfer momenta
}

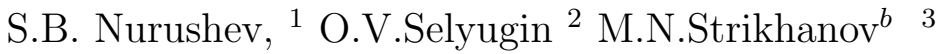

\begin{abstract}
The Coulomb-hadron interference effects are examined at small and large $t$. The methods for the definition of spin-dependent parts of hadron scattering amplitude are presented. The additional contributions to analyzing power $A_{N}$ and the double spin correlation parameter $A_{N N}$ owing to the electromagnetichadron interference are determined in the diffraction dip domain of highenergy elastic hadron scattering.
\end{abstract}

The energy region of RHIC in the fixed targed mode $(\mathrm{FT})\left(p_{L}=30 \div 250 \mathrm{GeV} / \mathrm{c}\right)$ at small transfer momenta is distinguished especially as the region where the second Reggeon contributions fall from an essential to an insignificant amount. The polarization phenomena determined at a low energy by the second Reggeon contributions also fall also very fast. Hence, in this domain the contributions into spin phenomena coming from the interference of the Coulomb and the hadron amplitudes play the important role [1]. On this background we must determine how fast fall in actually the spin-flip amplitudes of the second reggeons. Moreover, the high statistic and hence small errors can allow us to find the contributions of the possible non-falling spin flip amplitudes originated from an instanton or high twist interactions.

The definition of the structure of the high energy elastic hadron-hadron scattering amplitude at small angles is a topical problem [2]. Such quantities as the total cross

\footnotetext{
${ }^{1}$ Institute for High Energy Physics, 142284 Protvino, Moscow

${ }^{2}$ BLTP, JINR, 141980 Dubna, Russia; e-mail: selugin@thsun1.jinr.ru

${ }^{3}$ Moscow Engineering Physics Institute, Kashirrskoe Ave.31, 115409 Moscow, Russia
} 
section and the phase of the scattering amplitude are relevant to the fundamental relations of the strong interaction theory as, for example, the dispersion relations. At present we cannot calculate these parameters from the first principle or in the framework of the Perturbative Quantum Chromodynamics (PQCD), therefore they should be measured in experiment.

The differential cross section and spin parameters $A_{N}$ and $A_{N N}$ are defined as

$$
\begin{gathered}
\frac{d \sigma}{d t}=\frac{2 \pi}{s^{2}}\left(\left|\phi_{1}\right|^{2}+\left|\phi_{2}\right|^{2}+\left|\phi_{3}\right|^{2}+\left|\phi_{4}\right|^{2}+4\left|\phi_{5}\right|^{2}\right), \\
\left.A_{N} \frac{d \sigma}{d t}=-\frac{4 \pi}{s^{2}} \operatorname{Im}\left[\left(\phi_{1}+\phi_{2}+\phi_{3}-\phi_{4}\right) \phi_{5}^{*}\right)\right]
\end{gathered}
$$

and

$$
A_{N N} \frac{d \sigma}{d t}=\frac{4 \pi}{s^{2}}\left[\operatorname{Re}\left(\phi_{1} \phi_{2}^{*}-\phi_{3} \phi_{4}^{*}\right)+2\left|\phi_{5}\right|^{2}\right]
$$

in framework of usual helicity representation.

This information can be obtained from the precision measurement of the form of spin correlation parameters $A_{N}$ and $A_{N N}$ at small transfer momenta. In this domain the analyzing power $A_{N}$ is determined by the Coulomb-hadron interference effects. As we can calculate the Coulomb amplitude very precisely from the theory, we can obtain some information about the hadronic non-flip amplitude from this quantity.

If we know the parameters of the hadron spin non-flip amplitude, the measurement of the analyzing power at small transfer momenta helps us to find the structure of the hadron spin-flip amplitude. For that let us rewrite (2)

$$
\begin{aligned}
A_{N} \frac{d \sigma}{d t}= & 2\left[\left(I m F_{h}^{++} \operatorname{Re} F_{c}^{+-}+\operatorname{Im} F_{c}^{++} \operatorname{Re} F_{c}^{+-}-R e F_{h}^{++} I m F_{c}^{+-}\right.\right. \\
& \left.-\operatorname{Re} F_{c}^{++} I m F_{c}^{+-}\right)+\left(I m F_{h}^{++} R e F_{h}^{+-}-R e F_{c}^{++} I m F_{h}^{+-}\right. \\
& \left.\left.+\operatorname{Im} F_{c}^{++} \operatorname{ReF}_{h}^{+-}-\operatorname{ReF}_{h}^{++} I m F_{h}^{+-}\right)\right]
\end{aligned}
$$


where $F^{++}, F^{+-}, F^{--}$are the spin-non flip, spin-flip and spin double-flip amplitudes, correspondingly, with ordinary connection with helicity amplitudes, for example, $F^{++}=\left(\phi_{1}+\phi_{3}\right) / 2$. Let us denoted $P^{\prime}$ is the part of the analyzing power which can be calculated if we know $\sigma_{\text {tot }}, B, \rho$ for the non-flip amplitude. $\Delta P$ is the part depending on the hadron spin-flip amplitude.

$$
\Delta P=A_{N}^{\text {exper. }}-P^{\prime}
$$

Here $A_{N}^{\text {exper. }}$ is the measured value of the analyzing power. Let us factor out the term $F_{c}^{++}$from the numerator, the term $\left(F_{c}^{++}\right)^{2}$ from the denominator and multiply $\Delta P$ by $F_{c}^{++}$:

$$
A_{N}^{\prime}=(\Delta P) F_{c}^{++} \simeq 2 \frac{-I m F_{h}^{+-}+R e F_{h}^{+-}\left(I m F_{h}^{++} / R e F_{c}^{++}\right)}{1+\left(I m F_{h}^{++} / R e F_{c}^{++}\right)^{2}}
$$

So, at the point $t$ where $\left|I m F_{h}^{++}\right|=\left|R e F_{c}^{++}\right|$for the proton-proton scattering we have

$$
A_{N}^{\prime}=-R e F_{h}^{+-}-I m F_{h}^{+-}
$$

And, as $t \rightarrow 0$

$$
A_{N}^{\prime} \rightarrow-2 I m F_{h}^{+-}
$$

These calculations are compared with some model amplitude $F^{+-}$on Fig.1. at energies $\sqrt{s}=50$ and $500 \mathrm{GeV}$ (short and long dashed line).

Now, let us examine the hadron double-spin-flip amplitude which has not the kinematical zero as $t \rightarrow 0$. This amplitude plays an important role at low energies, where they exist owing to a spin-spin interaction and an unnatural parity exchange, and has a sufficiently large magnitude. The measurement of $A_{N N}$ at very small transfer momenta $\sim 10^{-4} \div 10^{-2} \mathrm{GeV}^{2}$ gives information about the structure of the double-spin flip amplitude and its energy dependence. For the double-spin 


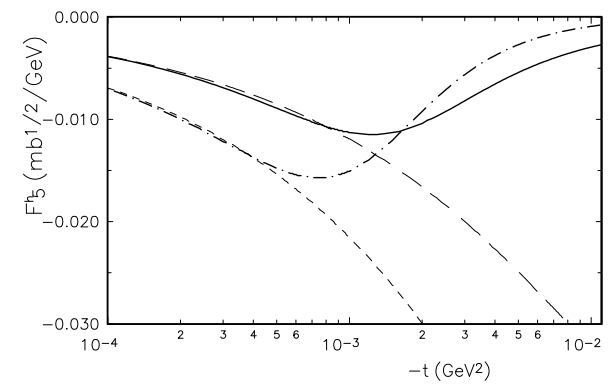

Figure 1: The calculated $A_{N}^{\prime} / 2$

correlation parameter $A_{N N}$ one can write:

$$
\begin{aligned}
A_{N N}(d \sigma / d t)= & 2 F_{c}^{++} F_{c}^{--}+F_{c}^{++} \operatorname{Re}\left(F_{h}^{--}\right)+\rho \operatorname{Im}\left(F_{h}^{++}\right) \operatorname{Re}\left(F_{h}^{--}\right) \\
& +\operatorname{Im}\left(F_{h}^{++}\right) \operatorname{Im}\left(F_{h}^{--}+2\left(F_{c}^{+-}\right)^{2}+2 F_{c}^{+-} \operatorname{Re}\left(F_{h}^{+-}\right)+2\left|F_{h}^{+-}\right|^{2}\right.
\end{aligned}
$$

Note, that

$$
2 F_{c}^{++} F_{c}^{--}+2\left(F_{c}^{+-}\right)^{2}=0
$$

so these contributions, significant separately, disappear.

If (3) is multiplied by the Coulomb amplitude, one can obtain in the range of small transfer momenta

$$
A_{N N}^{\prime}=A_{N N} F_{c}^{++} \simeq \frac{R e\left(F_{h}^{--}\right)+\operatorname{Im}\left(F_{h}^{--}\right) R+\rho\left[F_{c}^{--}+R e\left(F_{h}^{--}\right)\right] R}{1+R^{2}}
$$

where $R=\operatorname{Im}\left(F_{h}^{++}\right) / F_{c}^{++}$. So, at the point $t$, where $\left|\operatorname{Im}\left(F_{h}^{++}\right)\right|=\left|F_{c}^{++}\right|$, it gives

$$
A_{N N}^{\prime} \simeq 0.5\left[\operatorname{Re}\left(F_{h}^{--}\right)+\operatorname{Im}\left(F_{h}^{--}\right)\right] .
$$

And as $t \rightarrow 0$,

$$
A_{N N}^{\prime} \rightarrow \operatorname{Re}\left(F_{h}^{--}\right)
$$

These calculations are compared with some model amplitude $F_{h}^{--}$on Fig.2. at energies $\sqrt{s}=50$ and $500 \mathrm{GeV}$ (short and long dashed line). 


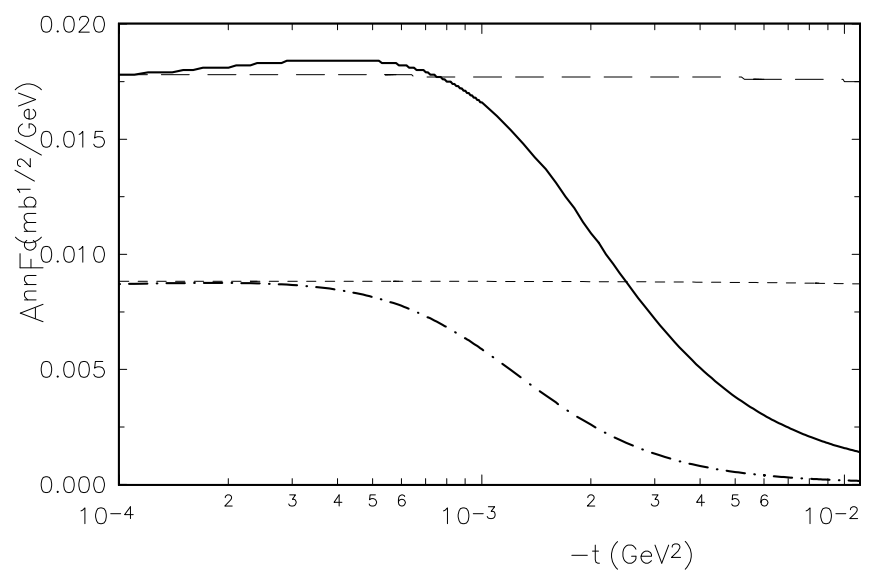

Figure 2: The calculated $A_{N N}^{\prime}$

Hence, the measurement of the double-spin correlation parameter at very small transfer momenta helps us to find the structure of the hadron double-spin-flip amplitude. Note that to find the point where $\left|\operatorname{Im}\left(F_{h}^{++}\right)\right|=\left|\operatorname{Re}\left(F_{c}^{++}\right)\right|$, one again needs know exactly the real part of the spin-non-flip amplitude.

So, we can conclude that the precise measurement of the spin correlation parameters $A_{N}$ and $A_{N N}$ at very small transfer momenta $-t \sim 10^{-4} \div 10^{-2} \mathrm{GeV}^{2}$ gives us a way for the definition of the parameters of the high energy hadron interaction. It allows us to reconstruct the structure of the high energy elastic scattering amplitude. Moreover, the precisely definition of the position of maximum of the analysing power permit us to define the value of the total cross section in an independent way. Hence, such a measurement is to be included in future research programs at HERA, RHIC and LHC.

At present, the spin effects owing to the Coulomb-nucleon interference (CNI) at very small transfer momenta are widely discussed in the aspect of future spin experiments at RHIC and LHC. These effects are worse understood in the domain 
of the diffraction dip. In many respects, this is due to the fact that we do not know the Coulomb-hadron interference phase for not small transfer momenta and its impact on the magnitude of the spin effects.

In papers [3, 4] the importance of CNI effects in the dip domain was pointed out. In [3] the polarization was calculated at sufficiently low energies with CNI-effect, but without taking accounted the phase of CNI and with grude approximation of the hadron non-flip spin amplitude.

The phase of the Coulomb-hadron interaction has been calculated and discussed by many authors [5, 6, 7] and has the form [8]

$$
\varphi(s, t)=\mp\left[\gamma+\ln (B(s, t)|t| / 2)+\ln \left(1+8 /\left(B(s, t) \Lambda^{2}\right)\right)+\nu_{c}\right.
$$

where $B(s, t)$ is the slope of the nuclear amplitude; $\Lambda$ is a constant entering into the dipole form factor and $\nu_{c}$ is an additional part coming from the pure Coulomb interaction and calculated in [8] for small $t$.

In [9], the phase $\nu_{c}$ of the pure Coulomb amplitude in the second Born approximation with the form factor in the monopole and dipole forms has been calculated in a wide region of $t$. It was shown that the behavior of $\nu_{c}$ at not small $t$ sharply differ from the behavior of $\nu_{c}$ obtained in [8]. Let us calculate the total phase factor that can be used in the whole diffraction range of elastic hadron scattering. The scattering amplitude in an eikonal representation separating the Coulomb and the hadron eikonals can be write:

$$
F(s, t)=F_{c}(s, t)+F_{h}(s, t)\left[1-\frac{1}{2 i F_{h}(s, t)} \int_{0}^{\infty} \chi_{c}(\rho)\left(1-e^{\chi_{h}(\rho, s)}\right) \rho d \rho J_{0}(\rho q)\right]
$$

Taking into account the pure Coulomb phase $\varphi_{c}$ we can rewrite (15) in terms of the phase factor

$$
F(s, t)=F_{c} e^{i \alpha\left(\varphi_{c h}+\varphi_{c}\right)}+F_{h}(s, t),
$$


with

$$
\varphi_{c}=\ln \frac{\lambda^{2}}{q^{2}}+\nu
$$

Here, $\lambda \rightarrow 0$ is the effective photon mass. Note that the divergent logarithmic term appears in the second Born approximation. Hence, if we take the Coulomb amplitude in first order, this term will be absent.

To calculate of the Coulomb part in the Coulomb-hadron eikonal, let us expand the nucleon form-factor in inverse powers of $\Lambda^{2}+q^{2}$ :

$$
\begin{aligned}
G^{2}\left(q^{2}\right) & \equiv\left[\frac{\Lambda^{2}}{\Lambda^{2}+q^{2}}\right]^{4} \\
& =1-\frac{q^{2}}{\Lambda^{2}+q^{2}}-\frac{q^{2} \Lambda^{2}}{\left(\Lambda^{2}+q^{2}\right)^{2}}-\frac{q^{2} \Lambda^{4}}{\left(\Lambda^{2}+q^{2}\right)^{3}}-\frac{q^{2} \Lambda^{6}}{\left(\Lambda^{2}+q^{2}\right)^{4}}
\end{aligned}
$$

Then, we obtain for the Coulomb part of the Coulomb-hadron eikonal:

$$
\begin{aligned}
\chi_{c}(\rho)= & -2 i\left[\alpha \left(K_{0}(\rho \lambda)-K_{0}(\rho \Lambda)-\frac{\Lambda \rho}{2 \Gamma(2)} K_{-1}(\rho \Lambda)-\frac{\Lambda^{2} \rho^{2}}{4 \Gamma(3)} K_{-2}(\rho \Lambda)\right.\right. \\
& \left.-\frac{\Lambda^{3} \rho^{3}}{8 \Gamma(4)} K_{-3}(\rho \Lambda)\right] .
\end{aligned}
$$

Here $K_{i}$ are MacDonald functions and $\Gamma(n)$ is a gamma function. The first term gives the eikonal without the nucleon form-factor and others give the corrections that play an important role when $t$ differs from zero. Using the logarithmic representation of $K_{0}$ we can take out from integral the first two terms, containing a divergences part and constant. The rest part of these two first terms in numerator, containing only the hadron eikonal, are canceled by the denominator. In result, the term with $\lambda$ is reduced with the same term of $\chi_{c}$ at calculation observable and the total phase factor is:

$$
\varphi(s, t)=\ln \frac{q^{2}}{4}+2 \gamma+\frac{1}{F_{h}(s, q)} \int_{0}^{\infty} \tilde{\chi}_{c}(\rho)\left(1-\exp \left(\chi_{h}(\rho, s)\right) J_{0}(\rho, q) d \rho,\right.
$$

with

$$
\tilde{\chi}_{c}(\rho)=2 \rho \ln \rho+2 \rho K_{0}(\rho \Lambda)\left[1+\frac{5}{24} \Lambda^{2} \rho^{2}\right]+\frac{\Lambda \rho}{12} K_{1}(\rho \Lambda)\left[11+\frac{5}{4} \Lambda^{2} \rho^{2}\right]
$$


The calculated $\varphi(s, t)(20)$ is an eikonal analog with taking account of the hadron form-factor of the expression obtained by West and Yennie [7] from the Feynman diagram.

If we take the Born term of the hadron amplitude in the Regge form

$$
F_{h}^{B}(s, q)=h \exp \left(-B(s) q^{2}\right)
$$

and only the first term of (20) that corresponds to the Coulomb amplitude without the form-factor, we obtain the ordinary representation for the total phase factor in the region of small transfer momenta

$$
\varphi \simeq \ln \frac{B(s) q^{2}}{2}+\gamma
$$

Our eikonal representation for the $\varphi(s, t)$ (20) is valid in a wide region of $t$. If we take the correct hadron scattering eikonal that describes the experimental differential cross section including the domain of the diffraction dip, we can calculate the $\varphi(s, t)$ for that region of $t$. Note, that the calculated term has a real and a nonsmall imaginary part. The phase factor can be very important for the calculation of spin correlation parameters with taking into account the electromagnetic-hadron interference effects in the domain of the diffraction dip. In this region, the imaginary part of the hadron scattering amplitude has a zero and these effects can be not small. Beforehand, we don't know how large the additional contributions will be and how the phase factor affects on magnitudes of the spin correlation parameters. The largest effects will be in the energy range where the diffraction minimum has a strictly defined form and, thus, the real part of the non-flip hadron amplitude will be small.

We can take, for example, the eikonal of the spin-non-flip hadron amplitude obtained in [1] that provides a quantitative description of high-energy experimental data:

$$
-\chi_{h}(s, b)=h_{1}(s) \exp \left(-\mu(s) \sqrt{b^{2}+b_{p}^{2}(s)}\right)\left[1-h_{2}(s) \exp \left(-\mu(s) \sqrt{b^{2}+b_{p}^{2}(s)}\right)\right]
$$


where $b_{p}(s)$ and $\mu(s)$ are the effective radius and mass, and calculate the coulombhadron phase-factor [10].

As this model is based on the crossing symmetry of the scattering amplitude, the parameters in this eikonal are complex and, hence, the obtained scattering amplitude has a real and an imaginary part. Using this eikonal we can calculate the total phase factor, for example, for $\sqrt{s}=50 \mathrm{GeV}$ (the first starting energy of RHIC). Note that our results depend weakly on the model for the hadron spin-non-flip eikonal. Different models should give the same $\sigma_{\text {tot }}, \rho(s)=\operatorname{Re} F(s, 0) / \operatorname{ImF}(s, 0)$ and differential cross sections in a wide region of energies and momenta transfer.

The value of the coulomb-hadron phase-factor in the domain of the diffraction dip is large in magnitude with a positive maximum of the real part at $-t=1.25 \mathrm{GeV}^{2}$ and a negative maximum. at $-t=1.45 \mathrm{GeV}^{2}$. The imaginary part has one negative maximum at the point of the minimum of the diffraction dip.

Let us calculate, using the obtained $\varphi(s, t)$, an additional contribution to the analyzing power $A_{N}$ and double spin correlation parameter $A_{N N}$ owing to the electromagnetic-hadron interference $A_{N}^{e m N}$ and $A_{N N}^{e m N}$ in the diffraction dip domain of the proton-proton elastic scattering. This contributions should be taken into account when we want to know the true size of the contribution to $A_{N}$ and $A_{N N}$ due to the hadron spin-flip amplitude. Let us again take the hadron spin-non-flip amplitude calculated in [11] and calculate the size of $A_{N}^{e m}$ in the diffraction dip domain for example, at energy HERA $\sqrt{s}=40 \mathrm{GeV}$. The calculated analyzing power is shown in Fig.3a. Its form is defined by the form of the diffraction minimum and the behavior of the imaginary part of the hadron spin-non-flip amplitude. As it is noted above, our result coincides qualitatively with the calculation of [3] up to the moment when the imaginary part of the hadron non-flip amplitude changes in sign and the polarization has a negative maximum. On the whole, the picture is similar to that of behavior of $\varphi$. The analysis shows that the impact of $\varphi$ leads in 


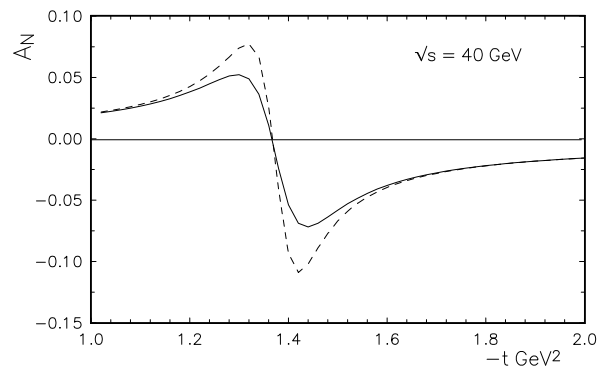

a)

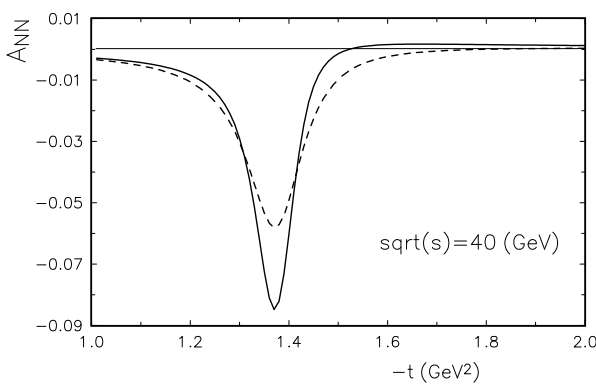

b)

Figure 3: The calculated a) $A_{N}^{e m N}$ and b) $A_{N N}^{e m N}$

the proton-proton scattering to the increase of the analyzing power approximately by $50 \%$ of the effect. Hence, if only this impact were negative, the size of $A_{N}^{e m N}$ becomes very small.

Now let us examine such an additional contribution to the double spin correlation parameter. It is clear that the large contribution to $A_{N N}^{e m N}$ comes from the interference of electromagnetic amplitudes $\phi_{1}^{e m}$ and $\phi_{2}^{e m}$. but in our case it is cancelled completely by the contribution of $2\left|\phi_{5}^{e m}\right|^{2}$ ).

So, we have the magnitude of $A_{N N}^{e m N}$ both dependent on the real part of the hadron spin-non-flip amplitude and $\phi_{2}^{e m}$. Our calculation of $A_{N N}^{e m N}$ is shown in Fig. $3 b$.

We obtain the negative non-small contribution which reduces the size of the double spin correlation parameter owing to the hadron-spin-flip amplitude. Of course, the form and size of $A_{N N}^{e m N}$ are mostly defined by the form and size of the diffraction minimum.

In spite of the large contribution of the hadron-spin-flip amplitude we can see that taking account of the electromagnetic amplitudes leads to visible changes in 
spin correlation effects. Note that in our case the part of the contribution of the interference electromagnetic and hadron-spin-non flip amplitude is reduced by terms of the interference between of the electromagnetic and hadron-spin-flip amplitude. On the whole, the spin correlation effects somewhat decrease and slightly change the positions of maxima.

Using the eikonal representation we obtain the terms corresponding to the electromagnetichadron interference in the second Born approximation taking into account the formfactors of interacting nucleons in a wide region of transfer momenta up to the diffraction dip domain. It allows us to calculate an additional contribution to the analyzing power $A_{N}$ and double spin correlation parameter $A_{N N}$ owing to the interference of the electromagnetic and hadron-spin-non-flip amplitudes. As a result, we obtain, in the domain of the diffraction minimum, not small spin correlation effects due to the interference of the spin-non-flip elastic scattering amplitude and the electromagnetic

spin-flip amplitude. The obtained energy dependence of $A_{N}^{e m N}$ and $A_{N N}^{e m N}$ shows that these contributions have to be taken into account when we extract the size and energy dependence of the hadron spin-flip amplitude from the experimental data on $A_{N}$ and $A_{N N}$ at high energies up to the top of RHIC energy.

\section{References}

[1] S.B. Nurushev et al.: In Proc. "Spin-93", Protvino, 1993, (1994) 321.

[2] O.V.Selyugin: Phys.Lett. B 333 (1994) 245.

[3] C. Bourrely, J. Soffer: Lett. Nuovo Cim. 19 (1977) 561.

[4] L.I. Lapidus: Particles \& Nuclei 9 (1978) 84.

[5] H. Bethe: Ann. Phys. 3190 (1958); L.I. Lapidus: Particles \& Nuclei 9 (1978) 84; N. H. Buttimore, E. Gotsman, E. Leader: Phys. Rev. D 35 (1987) 407. 
[6] M.M. Islam: Phys.Rev. 162 (1967) 1426.

[7] G. B. West, D. R. Yennie: Phis. Rev. 172 (1968) 1414

[8] R. Cahn: Zeitschr. fur Phys. C 15253 (1982) 253.

[9] O.V. Selyugin: Mod.Phys.Lett. A 11, (1996) 2317.

[10] O.V. Selyugin: Mod.Phys.Lett. A A 12 (1997) 1379.

[11] S. V. Goloskokov, S. P. Kuleshov, O. V. Selyugin: Zeit. fur Phys. C 50 (1991) 455. 